\title{
ENCRYPTED DATABASE INTEGRITY IN DATABASE SERVICE PROVIDER MODEL
}

\author{
Hakan Hacigümüş \\ Department of Information and Computer Science \\ University of California, Irvine, CA 92697, USA * \\ hakanh@acm.org
}

Bala Iyer

IBM Silicon Valley Lab.

San Jose, CA 95141, USA

balaiyer@us.ibm.com

\author{
Sharad Mehrotra \\ Department of Information and Computer Science \\ University of California, Irvine, CA 92697, USA \\ sharad@ics.uci.edu
}

\begin{abstract}
In the database service provider model, a service provider hosts client's data and allows access to data via a database language such as SQL. Database service provider model offers considerable benefits to organizations with data management needs. Yet, the model introduces many significant challenges, in particular that of data privacy and security. Ensuring the integrity of the database, which is hosted by a service provider, is a critical and challenging problem in this context. We propose an encrypted database integrity scheme, which allows the owner of the data to ensure the integrity of the database hosted at the service provider site, in addition to security of the stored data against malicious attacks.
\end{abstract}

Keywords: database, security, privacy, integrity, authentication, encryption, cryptography, e-service, service provider model

* Supported in part by an IBM Ph.D. Fellowship. This work was performed while the author was at IBM.

The original version of this chapter was revised: The copyright line was incorrect. This has been corrected. The Erratum to this chapter is available at DOI: 10.1007/978-0-387-35696-9_19 


\section{INTRODUCTION}

Database as a Service paradigm is introduced in [3]. Database service provider model (DSPM) is aimed at challenging the traditional model of data management followed by current organizations. The new model allows the organizations to outsource their data management services.

Among the others, data privacy and security are the most significant challenges in database service provider model. In DSPM the user data is stored at the service provider (ASP) site. The ASP stores the client's data and the client poses queries against that database and the ASP (or the server) responds back to the client with results of the queries. Most companies and individuals view their data as an asset. Therefore the owner of the data needs to be assured that the data is protected against malicious attacks from outside of ASP. The second and more challenging problem is privacy of the data when even the ASP itself is not trusted by the owner of the data. First problem is examined in [3] and the second one is studied in [2], which explores how SQL queries can be executed over encrypted data.

In this paper, we look at another important issue that arises in the context of the second problem stated above. Although the client's data is protected against both outsiders and the ASP with data encryption techniques, how can the client ensure the integrity of the database and results of the queries reported back by the ASP?

The integrity problem has two dimensions. First, when the client receives a record from the sever, how can the client ensure the integrity of the record? That is, how can the client verify that the data has not been changed in an unauthorized way? Second, when the client receives set of answers for the query, how can the client be certain that all of the records, which are qualified by the query are included in the results?

These problems may arise both from malicious or non-malicious circumstances. Malicious or intentional threats may originate from misbehaving server or some other adversary who breaks into the system. Non-malicious or unintentional threats can also have many sources. One example for those is system failures. ASP may experience a system breakdown and may not be able to recover all user data from on-line and/or archive sources. In those cases, the client does not have any control mechanism to detect integrity of the original data. To address these issues, we propose two-level encrypted database integrity scheme, which consists of Record Level Integrity and Table Level Integrity concepts.

The rest of the paper is organized as follows. Section 2 presents DSPM architecture. Section 3 describes encrypted database storage model. Section 4 presents our solution to encrypted database integrity in DSPM. We conclude the paper in Section 5. 


\section{DATABASE APPLICATION SERVICE PROVIDER MODEL}

The system we use in this study is based on the architecture proposed and described in [2]. It is comprised of three fundamental entities. A user poses the query to the client. A server is hosted by the service provider that stores the encrypted database. The encrypted database is augmented with additional information, which we call the index, that allows certain amount of query processing to occur at the server without jeopardizing data privacy. A client stores the data at the server. Client ${ }^{1}$ also maintains metadata for translating user queries to the appropriate representation on the server, and performs post-processing on server query results.

\section{ENCRYPTED DATABASE STORAGE MODEL}

In this section we first summarize the model, which is used to 1) create the metadata at the client site and 2) store the encrypted database at the server. ${ }^{2}$ Following this we introduce our extensions to the model to implement data integrity techniques.

For each relation $R\left(A_{1}, A_{2}, \ldots, A_{n}\right)$, we store on the server an encrypted relation: $R^{S}$ (etuple, $\left.A_{1}^{S}, A_{2}^{S}, \ldots, A_{n}^{S}\right)$, where the attribute etuple stores an encrypted string that corresponds to a tuple in relation $R$. Any deterministic encryption technique, such as DES [1], can be used to compute etuples. Each attribute $A_{i}^{S}$ corresponds to the index for the attribute $A_{i}$. For example, consider a relation emp below that stores information about employees.

\begin{tabular}{|l|l|l|l|l|}
\hline eid & ename & salary & addr & did \\
\hline \hline 23 & Tom & $70 \mathrm{~K}$ & Maple & 40 \\
\hline 860 & Mary & $60 \mathrm{~K}$ & Main & 80 \\
\hline 320 & John & $50 \mathrm{~K}$ & River & 50 \\
\hline 875 & Jerry & $75 \mathrm{~K}$ & Hopewell & 110 \\
\hline 50 & Bill & $70 \mathrm{~K}$ & Pine & 80 \\
\hline 875 & Sarah & $80 \mathrm{~K}$ & Oak & 80 \\
\hline
\end{tabular}

Table 1 Relation emp : Information about employees

The $e m p$ table is mapped to a corresponding table at the server $e m p^{S}$ as given in Table $2 .^{3}$

\footnotetext{
${ }^{1}$ Often the client and the user might be the same entity.

${ }^{2}$ We will not repeat here all of the details of storage model, since it is discussed thoroughly in [2]. Rather, we only provide necessary notations to explain the constructs we develop in this work.

${ }^{3}$ Tuple identifier (TID) column is used for presentation purposes only and it is not actually part of the table. We will use TIDs to illustrate the examples more clearly in the following sections.
} 


\begin{tabular}{|c|c|c|c|c|c|c|}
\hline TID & etuple & eid $^{S}$ & ename $^{S}$ & salary $^{S}$ & addr $^{S}$ & did $^{S}$ \\
\hline \hline 1 & $1100110011110010 \ldots$ & 2 & 19 & 81 & 18 & 2 \\
\hline 2 & $1000000000011101 \ldots$ & 4 & 31 & 59 & 41 & 5 \\
\hline 3 & $1111101000010001 \ldots$ & 7 & 7 & 59 & 22 & 4 \\
\hline 4 & $1010101010111110 \ldots$ & 4 & 71 & 81 & 20 & 6 \\
\hline 5 & $1110111010101110 \ldots$ & 2 & 71 & 81 & 32 & 5 \\
\hline 6 & $1011110110111110 \ldots$ & 4 & 71 & 81 & 40 & 5 \\
\hline
\end{tabular}

Table 2 Encrypted version $e m p^{S}$ of relation $e m p$

Creation of index values involves three fundamental functions:

Partition Functions divide domain of values of attribute $A_{i}$ in relation $R$ into partitions (or buckets) $\left\{p_{1}, \ldots, p_{k}\right\}$. Identification Functions, called ident, assign an identifier ident $_{R . A_{i}}\left(p_{j}\right)$ to each partition $p_{j}$ of attribute $A_{i}$. Mapping Functions map a value $v$ in the domain of an attribute to the identifier of the partition to which $v$ belongs. For example, the following shows some values of the mapping function for attribute emp.eid based on the partitioning given in Figure 1: $\operatorname{Map}_{\text {emp.eid }}(23)=2, \operatorname{Map}_{\text {emp.eid }}(860)=4$, and Map emp.eid $(875)=4$.

\begin{tabular}{lllllllllll}
$L$ & 2 & $\perp$ & 7 & 1 & 5 & 1 & 1 & 1 & 4 & L \\
\hline & & 200 & & 400 & & 600 & & 800 & & 1000
\end{tabular}

Figure 1 Partition and identification functions of emp.eid

\subsection{MAPPING CONDITIONS}

In this section we study how to translate specific query conditions in operations to corresponding conditions over the server-side representation. This translation function is called $M a p_{\text {cond }}$.

We include two types of conditions; namely, Attribute = Value and Attribute1 = Attribute2, which will be enough to demonstrate our techniques for data integrity in our discussion here. ${ }^{4}$

Attribute $=$ Value: The mapping is defined as follows: $\operatorname{Map}_{\text {cond }}\left(A_{i}=v\right) \Rightarrow$ $A_{i}^{S}=\operatorname{Map}_{A_{i}}(v)$. For instance, consider the emp table above, we have: $M a p_{\text {cond }}(e i d=860) \Rightarrow e i d^{S}=4$, since $e i d=860$ is mapped to 4 by the mapping function of this attribute.

${ }^{4}$ Full discussion of mapping conditions can be found in [2]. 
Attribute1 = Attribute2: The following is the translation:

$$
\operatorname{Map}_{\text {cond }}\left(A_{i}=A_{j}\right) \Rightarrow \bigvee_{\varphi}\left(A_{i}^{S}=\operatorname{ident}_{A_{i}}\left(p_{k}\right) \wedge A_{j}^{S}=\operatorname{ident}_{A_{j}}\left(p_{l}\right)\right)
$$

where $\varphi$ is $p_{k} \in \operatorname{partition}\left(A_{i}\right), p_{l} \in \operatorname{partition}\left(A_{j}\right), p_{k} \cap p_{l} \neq \emptyset$.

\begin{tabular}{|c|c|c|c|}
\hline Partitions & Ident $_{\text {emp.did }}$ & Partitions & Ident $_{\text {mgr.did }}$ \\
\hline \hline$[0,100]$ & 2 & {$[0,200]$} & 9 \\
\hline$(100,200]$ & 4 & $(200,400]$ & 8 \\
\hline$(200,300]$ & 3 & & \\
\hline$(300,400]$ & 1 & & \\
\hline
\end{tabular}

For instance, the table above shows the partition and identification functions of two attributes emp.did and mgr.did. Then condition $C$ : emp.did = $m g r . d i d$ is translated to the following condition $C^{\prime}$ :

$$
\begin{array}{r}
\left(e m p^{S} \cdot d i d^{S}=2 \wedge m g r^{S} \cdot d i d^{S}=9\right) \vee\left(e m p^{S} \cdot d i d^{S}=4 \wedge m g r^{S} \cdot d i d^{S}=9\right) \\
\vee\left(e m p^{S} \cdot d i d^{S}=3 \wedge m g r^{S} \cdot d i d^{S}=8\right) \vee\left(e m p^{S} \cdot d i d^{S}=1 \wedge m g r^{S} \cdot d i d^{S}=8\right)
\end{array}
$$

\subsection{OBSERVATIONS ABOUT MAPPING CONDITIONS}

The above described $M a p_{\text {cond }}$ function is used to translate a client's SQL query into a query that can execute at the server. In [2], it is shown that the resulting server side query returns to the client superset of tuples that qualify the query conditions. The client, after decrypting the tuples, can filter out tuples that does not qualify thereby returning the actual answers. We make note of one property of the above defined mechanism to map conditions in original client query to its server side translation - each condition in the original query is translated into a server side condition, which is in disjunctive normal form and each atomic condition in the query is in the form of Attribute=Value. We will make use of this property when we try to ensure table level integrity later.

\subsection{PARTITION COUNTERS}

As it is described, the client maintains metadata information for the partitions and partition ids of the attributes in the database. We propose, in addition to those, metadata contains partition counters for each partition of an attribute. This provides number of current records in each partition. Partition counters are updated upon insertion/deletion operations to the database. We will utilize this information to implement table level security mechanisms. As an example, for salary attribute of $e m p$ table the client stores the following metadata. 


\begin{tabular}{|c|c|c|}
\hline Partitions & Ident $_{\text {emp.salary }}$ & PartitionCounter \\
\hline \hline$[0 \mathrm{~K}, 20 \mathrm{~K}]$ & 21 & 0 \\
\hline$(20 \mathrm{~K}, 40 \mathrm{~K}]$ & 43 & 0 \\
\hline$(40 \mathrm{~K}, 60 \mathrm{~K}]$ & 59 & 2 \\
\hline$(60 \mathrm{~K}, 80 \mathrm{~K}]$ & 81 & 4 \\
\hline
\end{tabular}

\section{ENCRYPTED DATABASE INTEGRITY}

There are two entities the client needs to possess to verify the correctness or integrity of the results sent by the server, 1) Authenticity of the records and 2) Number of records that are supposed to be sent for a particular query. We call the former as Record Level Integrity and the latter as Table Level Integrity.

Data integrity, in general, can be defined as a property, which guarantees that the data has not been manipulated in an unauthorized manner since the time it was created by an authorized source. Manipulation detection codes (MDCs) provide this level data integrity in combination with data encryption $[4,5]$. MDCs are one-way collision resistant hash functions that provide a representative image or hash of a message. We need to expand this definition to satisfy data integrity requirements in database service provider model. This definition only provides data integrity for individual records stored in the database at the server site. In addition to that, we want to ensure that the server replies with correct set of records to the client.

Record level integrity represents that the content of a record has not been manipulated in an unauthorized manner. Table level integrity signifies that the server has reported all of the records, which qualify a particular query posed by the client.

To provide record level integrity we propose a scheme based on Record Integrity Codes (RICs). RICs are specially computed representative images for each record with certain security and uniqueness measures.

We present group of methods to implement table level integrity. The crux of those methods is to determine number of expected records for a given query and we employ the storage and query execution models presented in [2] as the basis to develop them.

\subsection{RECORD LEVEL INTEGRITY}

Figure 2 shows the procedure that provides record level data integrity. The client has a record $r$ that will be inserted into database, which is maintained by the application service provider or simply the server. The client first computes the hash code of the record $H=h(r)$ by using hash function $h$, which produces Record Integrity Code (RIC). This can be any algorithm, which satisfies the security requirements given before. Here we use MDCs for this purpose. After this step, the client concatenates the hash code $H$ to the origi- 


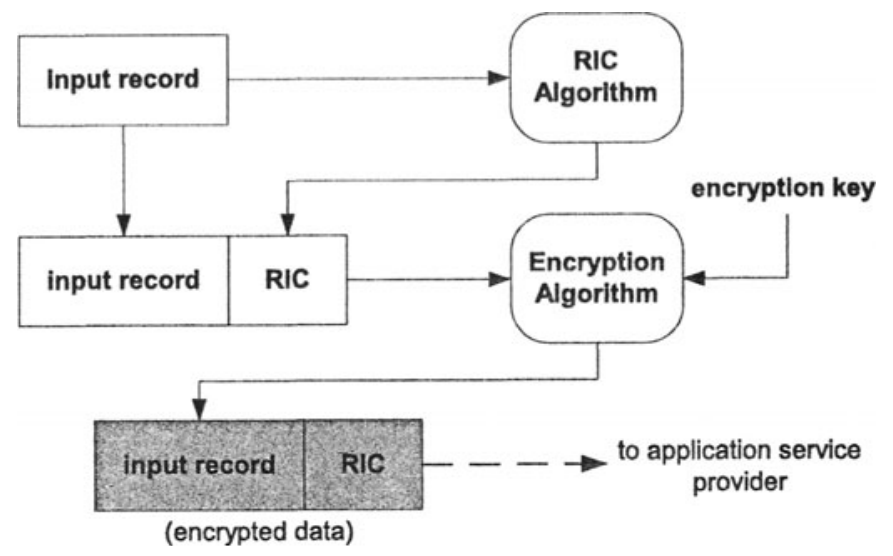

Figure 2 Record level integrity with RICs

nal record text $r$ and encrypts them together by using any deterministic encryption algorithm $E$ with secret key $k$, i.e., the client computes ciphertext $C=E_{k}(r \| h(r))$. The client inserts ciphertext $C$ as etuple into the database.

Whenever the client requests a record, the server sends back corresponding etuple in encrypted form. To verify the integrity of the record, the client first decrypts the etuple recovering $r^{\prime}$ and $H^{\prime}$, which is RIC, parts. Since only the client has the secret key $k$ for encryption algorithm no one else can decrypt. Then the client independently computes $h\left(r^{\prime}\right)$ of received record $r^{\prime}$ and compares that with hash code $H^{\prime}$. If they are equivalent, this verifies that the received record is authentic and has data integrity, i.e., has not been manipulated in an unauthorized manner.

Timestamping. If the client has two tuples, which have exact same content then the computed hash codes and RICs will be same for those. This is not the uncommon case for database applications. If the client is not able to differentiate those two records, it may lead to an integrity problem. In the case of data loss, for example, the server can easily duplicate the existing records and insert those replicas into the database.

We use timestamping to address this issue. Whenever the client inserts a record into the database, it first appends the timestamp $T S$ value to the input record $r$ and computes hash code of this augmented string and encrypts them together, to be exact, $C=E_{k}(r\|T S\| h(r \| T S))$.

When the client receives set of records from the server, upon decryption, the client can compare the $T S$ values of the records. Equivalent $T S$ values would surface the existence of spurious records in the result. 


\subsection{TABLE LEVEL INTEGRITY}

In this section we study how to verify query results returned by the server when the query has conditions. We referred to this as Table Level Integrity. When the query does not have any conditions in the text, then checking for integrity of the answer is straightforward as the query is expected to return all of the records available in the table or tables mentioned in the query. This implies returning all possible partitions defined for the table. Since the client maintains the count information for all partitions, it is always possible to check the correctness of the number of tuples returned by the server.

The more complicated case is, however, the queries with conditions. The client should be able to verify that all of the tuples, which satisfy the query conditions, have been returned by the server. This involves determining the correct number of expected tuples $S$ from the server. Combining this measure with record level integrity, the client ensures the authenticity and integrity of the query result sent by the server.

Let us consider how the client can determine correct number of expected tuples $S$ in the existence of query condition. We showed that all of the conditions in the original query are converted into disjunctive normal form and all of the atomic conditions are in the form of Attribute=Value. To demonstrate how the client can ensure the table level integrity, we first consider the atomic conditions characterized as Attribute=Value and show how the number of expected tuples can be determined by the client.

Attribute $=$ Value: In this case, first we have to identify the partition $p$ in which the value $v$ falls. This is provided by Map function. Then the number of expected tuples $S$ is the number of tuples in that partition. This information is provided in the metadata information stored at the client. Thus, $S=|p|=$ $\left|\operatorname{Map}_{A}(v)\right|$.

Example 4.1. For example, consider the relation emp given in Table 1 and a query condition $C:$ salary $=65 \mathrm{~K}$. First, this condition is translated to encrypted data model by using partitioning information, thus $\operatorname{Map}_{\text {salary }}(65 K)=$ 81. This follows that translated condition $C^{\prime}:$ salary $^{S}=81$. By using partition counts information we can find the number of expected tuples $S=\left|p_{81}\right|=$ 4 .

Next we explain how the client can ensure the integrity of the query answers when the query has arbitrary conditions, i.e., the query has the combinations of those conditions.

Conjunction of atomic conditions: Since in conjunctive form, all conditions are connected with AND operator, if the client can ensure that all of the tuples have been received for only one of the conditions, this would be enough to verify the query answer returned by the server. Hence, the client distin- 
guishes one of the conditions and requests all partitions, which satisfy the distinguished condition, from the server regardless of the other conditions. If there are any, all of the tuples, which constitute the answer of the query, definitely lie in the partitions of the distinguished condition. Therefore, upon decryption of etuples the client can extract all of the tuples that satisfy the conjunction.

Next question is how does the client choose the distinguished condition? Any of the conditions may be selected. We choose a condition whose partitions, which satisfy the condition, contain the least number of tuples at the server side since this reduces post-processing at the client side and network communication. Formally:

If we have a conjunction $C$ over set of attributes $A_{i}$ and set of values $v_{i}$ : $1 \leq i \leq n$ given as; $C: A_{1}=v_{1} \wedge A_{2}=v_{2} \wedge \ldots \wedge A_{n}=v_{n} \Rightarrow S=$ $\min _{i}\left\{\left|p_{1}\right|, \ldots,\left|p_{n}\right|\right\}=\min _{i}\left\{\left|\operatorname{Map}_{A_{1}}\left(v_{1}\right)\right|, \ldots,\left|\operatorname{Map}_{A_{n}}\left(v_{n}\right)\right|\right\}$

Consider the following example that illustrates this procedure:

Example 4.2. Let the conjunction be $C:$ salary $^{S}=81 \wedge \operatorname{did}^{S}=5$ and consider the relation $e m p^{S}$ given in Table 2. Then the result of the query $R$ (with TID) should be $R=\{5,6\}$. For the first condition $c_{1}:$ salary ${ }^{S}=81$, corresponding partition $p_{1}=81$ and $\left|p_{1}\right|=4$. Similarly for $c_{2}: d i d^{S}=5$, corresponding partition $p_{2}=5$ and $\left|p_{2}\right|=3$. Thus $c_{2}$ is selected as the distinguished condition. Therefore if the client wishes to verify the integrity of the result of the query, it requests all of the tuples that satisfies $c_{2}$. This should produce set of tuples $\{2,5,6\}$ and the client knows the number of tuples should be returned for the $p_{2}=5$. Upon decryption of etuples the client can filter out $\mathrm{TID}=2$ and reach the result of $\{5,6\}$ with integrity guarantee.

Disjunction of atomic conditions: In disjunctive form, all conditions are connected with OR operator. Therefore, what client needs to verify is whether correct number of tuples have been received for each of the conditions. As the client receives the tuples, it can count the number of tuples that fall in particular partitions, which are required to be retrieved for each of the atomic conditions. We call these numbers as receive counts. Obviously, in this case, number of expected tuples $S$ is defined as a set of receive counts $s_{i}$, which are the number of expected tuples for each corresponding partition $p_{i}$. After this, the client can compare receive counts with the actual number of tuples stored at the server for all partitions, which is provided by the metadata information stored at the client, to check the correctness. Once the client validate that all of the tuples in the partitions have been received, with the record level integrity measures, the client can be sure that the query result is correct. More formally we can give the notation for disjunction of the conditions as follows:

If we have a disjunction $D$ over set of attributes $A_{i}$ and set of values $v_{i}$ : $1 \leq i \leq n$ given as; 
$D: A_{1}=v_{1} \vee A_{2}=v_{2} \vee \ldots \vee A_{n}=v_{n} \Rightarrow S=\left\{s_{i}\left|s_{i}=\right| p_{i} \mid\right\}$, where $p_{i}$ is corresponding partition for condition $d_{i}$.

Consider the following example that illustrates this procedure:

Example 4.3. Let the disjunction be $D:$ salary $^{S}=81 \vee d i d^{S}=5$ and consider the relation $e m p^{S}$ given in Table 2 . Then the result of the query $R$ (with TID) should be $R=\{1,2,4,5,6\}$. For the first condition $d_{1}:$ salary ${ }^{S}=81$, corresponding partition $p_{1}=81$ and $\left|p_{1}\right|=4$. Similarly for $d_{2}: d i d^{S}=5$, corresponding partition $p_{2}=5$ and $\left|p_{2}\right|=3$. This information is already stored at the client site. Thus, upon decryption, the client can expect receive counts as 4 and 3 for partition $p_{1}=81$ and $p_{2}=5$ respectively. The client can detect incorrect results by comparing these numbers; receive counts, with the partition counts.

\section{CONCLUSIONS}

We studied the crucial problem of encrypted database integrity in the context of database service provider model. We proposed two-level encrypted database integrity scheme, which consists of Record Level Integrity and Table Level Integrity concepts, as a solution to this problem. Our scheme is combined with encrypted database storage model. Consequently, resultant system provides security of the stored data against malicious attacks as well as database integrity features, which ensure the authenticity and validity of the data stored at the service provider site.

\section{References}

[1] DES. Data Encryption Standard. FIPS PUB 46, Federal Information Processing Standards Publication, 1977.

[2] H. Hacıgümüş, B. Iyer, C. Li, and S. Mehrotra. Executing SQL over Encrypted Data in Database-Service-Provider Model. In Proc. of ACM SIGMOD, 2002.

[3] H. Hacıgümüş, B. Iyer, and S. Mehrotra. Providing Database as a Service. In Proc. of ICDE, 2002.

[4] D.R. Menezes, P.C. van Oorschot, and S.A. Vanstone. Handbook of Applied Cryptography. CRC Press, 1997.

[5] D.R. Stinson. Cryptography: Theory and Practice. CRC Press, 1995. 
CONTRIBUTED SHORT PAPERS 